\title{
A small molecule modulating monounsaturated fatty acids and Wnt signaling confers maintenance to induced pluripotent stem cells against endodermal differentiation
}

\author{
Vahid Hosseini 1,2,3 (10, Ashkan Kalantary-Charvadeh ${ }^{4}$, Maryam Hajikarami ${ }^{5}$, Parisa Fayyazpour ${ }^{3}$,
} Reza Rahbarghazi ${ }^{2,6}$, Mehdi Totonchi $^{7}$ and Masoud Darabi $2,3,8^{*}$

\begin{abstract}
Background: Stearoyl-coenzyme A desaturase 1 (SCD1) is required for de novo synthesis of fatty acids. Through the fatty acid acylation process, this enzyme orchestrates post-translational modifications to proteins involved in cell development and differentiation. In this study, we used biochemical methods, immunostaining, and covalent labeling to evaluate whether a small molecule modulating unsaturated fatty acids can influence the early endodermal differentiation of human-induced pluripotent stem cells (iPSCs).

Methods: The hiPSCs were cultured in an endoderm-inducing medium containing activin A and defined fetal bovine serum in the presence of an SCD1 inhibitor at different time points. The cell cycles and the yields of the three germ layers (endoderm, mesoderm, and ectoderm) were assessed using flow cytometry. The expression of endoderm and pluripotency markers and the expressions of Wnt signaling pathway proteins were assessed using western blotting and RT-PCR. Total protein acylation was evaluated using a click chemistry reaction.

Results: When SCD1 was inhibited on the first day, the population of cells with endodermal features decreased at the end of differentiation. Moreover, early SCD1 inhibition preserved the properties of hiPSCs, preventing their shift toward mesodermal or ectodermal lineage. Also, first-day-only treatment of cells with the SCD1 inhibitor decreased $\beta$-catenin gene expression and the intensity of fluorescent emission in the click chemistry assay. The cells were effectively rescued from these effects by cotreatment with oleate. Late treatment with the inhibitor in the two subsequent days of endoderm induction did not have any significant effects on endoderm-specific markers or fluorescent intensity. Reproducible results were also obtained with human embryonic stem cells.

Conclusion: The small molecule SCD1 inhibitor attenuates the Wnt/ $\beta$-catenin signaling pathway, conferring the maintenance of hiPSCs by opposing the initiation of endoderm differentiation. The immediate requirement for SCD1 activity in the endoderm commitment of pluripotent stem cells may be of importance in disorders of endodermderived organs and dysregulated metabolism.
\end{abstract}

Keywords: Germ layers, Desaturation, Pluripotent stem cells, Post-translational modification, Wnt signaling pathway, Wnt3a protein

\footnotetext{
${ }^{*}$ Correspondence: darabim@tbzmed.ac.ir

${ }^{8}$ Department of Internal Medicine IV, Heidelberg University Hospital, Heidelberg, Germany

Full list of author information is available at the end of the article
}

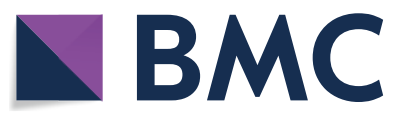

The Author(s) 2021. Open Access This article is licensed under a Creative Commons Attribution 4.0 International License, which permits use, sharing, adaptation, distribution and reproduction in any medium or format, as long as you give appropriate credit to the original author(s) and the source, provide a link to the Creative Commons licence, and indicate if changes were made. The images or other third party material in this article are included in the article's Creative Commons licence, unless indicated otherwise in a credit line to the material. If material is not included in the article's Creative Commons licence and your intended use is not permitted by statutory regulation or exceeds the permitted use, you will need to obtain permission directly from the copyright holder. To view a copy of this licence, visit http://creativecommons.org/licenses/by/4.0/. The Creative Commons Public Domain Dedication waiver (http://creativeco mmons.org/publicdomain/zero/1.0/) applies to the data made available in this article, unless otherwise stated in a credit line to the data. 


\section{Introduction}

Human-induced pluripotent stem cells (hiPSCs) are directly generated from adult somatic cells by reprogramming procedure. It has been suggested that hiPSCs have a great potential to commit into endodermal, mesodermal, and ectodermal cell lineages [1]. The existence of unique pluripotencies, high proliferation rates, lack of unwanted immunological reactions, and lack of ethical concerns make hiPSCs valuable biological tools in the area of regenerative medicine [2]. Furthermore, hiPSCs can be used in biomedical science for applications such as drug screening, finding the genetic basis of diseases, and investigating molecular mechanisms that regulate differentiation [3-6].

Monounsaturated fatty acids (MUFAs) are essential precursors for building cell structures and bioactive lipids [7]. Of note, the endoplasmic reticulum enzyme stearoylcoenzyme A desaturase 1 (SCD1) converts saturated fatty acids (SFAs) into MUFAs by adding a double bond between carbons 9 and 10, hence regulating the ratio of MUFAs to SFAs in cells $[8,9]$. Wnt ligand proteins are the most well-known biomolecules to undergo post-translational modification by addition of MUFAs. This process involves Porcupine (Porcn) catalytic activity, which appends MUFAs, particularly palmitoleate, onto the Ser209 residue of Wnt proteins [10]. It has been indicated that there is a close association between Wnt proteins and MUFAs. For instance, Wnt protein activation, intracellular and extra-cellular transportation, and binding to cell surface receptors are dependent on MUFA residues. In previous studies, the critical role of Wnt3a, as the main member of the Wnt family, has been documented in the niche of stem cells [11-13]. Like other members of the Wnt cascade, Wnt3a can transfer signaling to downstream effectors, such as the $\beta$-catenin/canonical pathway after MUFA-mediated interaction with cell surface receptors. The synergic effect of Wnt3a and activin A was shown in the differentiation of endoderm-derived organs $[14,15]$. In human embryonic stem cells (hESCs) and in induced pluripotent stem cells of mice (miPSCs), the activity of SCD1 supports cell growth and survival [16]. The in vitro suppression of SCD1 can lead to the selective elimination of pluripotent cells [17]. Interestingly, the injection of miPSCs and hESCs pre-treated with an SCD1 inhibitor reduced anaplastic changes and teratoma formation in immunosuppressed mice. Moreover, this treatment can improve stem cell safety after cardiac transplantation $[17,18]$. As a correlate, these findings confirm the crucial role of SCD1 in stem cell bioactivity and functional fate acquisition. High intracellular rates of SCD1 have been reported in endoderm-derived tissues, such as liver and pancreas tissue. Similarly, SCD1 content is also relatively high in some non-endodermal tissues such as adipose tissue, indicating SCD1's tissue- and lineage-dependent activity $[19,20]$. Furthermore, SCD1 activity in stem cells is relevant to the characteristics of adipogenic differentiation [21]. Previously, our research group showed the importance of SCD1 products in the hepatic differentiation of hiPSCs [22], which coincided with in vivo data [23]. Considering these findings, one could suggest that SCD1 products not only can enter the metabolic pathways but are also potentially involved in the signaling pathways related to cell differentiation.

There is no report to date assessing the kinetic effect of the small molecules targeting SCD1 during the endodermal differentiation of pluripotent stem cells (PSCs). In this study, we used biochemical methods, acylation assay, and immunostaining to evaluate whether the dynamics of SCD1 activity are important in the differentiation of hiPSCs into endodermal lineages. According to the results, small molecule SCD1 inhibitors can support the maintenance of hiPSCs by postponing endodermal differentiation.

\section{Materials and methods Materials}

All cell culture materials were obtained from Gibco (USA) unless otherwise specified.

\section{Primary mouse embryonic fibroblasts isolation}

Primary mouse embryonic fibroblasts (MEFs) were isolated by a mechanical technique and characterized as described in our previous study [24]. In short, embryonic fibroblasts with the passage number 1-5 were commonly proliferated in a culture flask pre-coated with $0.2 \%$ gelatin (Sigma, Germany) containing $10 \mathrm{ml}$ of Dulbecco's minimum eagle's medium (DMEM; Gibco, USA) and $10 \%$ fetal bovine serum (FBS). Before stem cell culture, MEFs were treated with $10 \mu \mathrm{g} / \mathrm{ml}$ Mitomycin C (Abcam, $\mathrm{UK}$ ) for $3 \mathrm{~h}$ to inhibit cell proliferation.

\section{Stem cell culture and expansion}

The established normal R1-hiPSC and hESC XX Royan H1 cell lines [25] were used in this study. The PSCs were characterized by stemness gene expression [25]. The PSCs culture media was composed of 75\% DMEM/ F12 supplemented with $20 \%$ knockout serum replacement, $1 \%$ non-essential amino acids, $2 \mathrm{mM} \mathrm{L}$-glutamine (Sigma, Germany), 0.1\% $\beta$-mercaptoethanol (Merck, Germany), $1 \%$ insulin/transferrin/selenium solution, and $12 \mathrm{ng} / \mathrm{ml}$ basic human recombinant FGF (b-FGF) (Royan, Iran). The standard cultures were split at the ratio of $1: 5$ every 7-10 days. 


\section{Endoderm differentiation}

Upon 70-80 confluence, hiPSCs were detached using $1 \mathrm{mg} / \mathrm{ml}$ Collagenase Type IV (Stem Cell Technologies, Canada) and dissociated into single cells by gentle pipetting. The cells were washed with phosphate-buffered saline (PBS) and suspended in fresh ESCs culture media containing $100 \mathrm{ng} / \mathrm{ml}$ of b-FGF, and then transferred on Matrigel- (Sigma, Germany) coated $60 \mathrm{~mm}$ plates. The next day, the medium was replaced with the differentiation medium containing RPMI-1640 with $100 \mathrm{ng} / \mathrm{ml}$ activin A (BioLegend, USA) with varying concentrations of defined-FBS (D-FBS). The concentrations of D-FBS were set to 0\% (Day 0), 0.2\% (Day 1), and $2 \%$ (Day 2 and 3 ). The cells were harvested and used for analyses at the fourth $24 \mathrm{~h}$ (Day 4) [26].

\section{Targeting SCD1 by small molecule inhibitor}

Chemical inhibition of SCD1 was performed using the specific inhibitor of SCD1 CAY10566 (Cayman Chemical, USA). Cells were treated with SCD1 inhibitor for $24 \mathrm{~h}$ at Days 0,1 , or 2 of the differentiation procedure. In our previous study [22], the possible toxicity of the inhibitor was determined using Trypan Blue exclusion and sulforhodamine B assays. Based on that, possible toxicity was excluded using $25 \mathrm{nmol} / \mathrm{l}$ CAY10566 [22]. The rescue experiments were performed using $50 \mu \mathrm{M}$ oleate.

\section{Evaluation of cell viability}

An annexin V-FITC/propidium iodide (PI) apoptosis detection kit (eBioscience, USA) was used for the apoptosis/necrosis analysis. After completion of the endodermal differentiation procedure, cells were detached with collagenase IV and washed once with PBS. The cells were suspended in the binding buffer (1X), incubated with $5 \mu \mathrm{l}$ annexin-V for $10 \mathrm{~min}$, and washed once with binding buffer. The cells were re-suspended again in $200 \mu \mathrm{l}$ of binding buffer followed by the addition of $10 \mu \mathrm{l}$ of propidium iodide. The percentage of apoptosis and necrosis was determined using a flow cytometer (Miltenyi Biotec, USA) and results were analyzed using flow Jo 7.6.1 software (Tree Star, USA).

\section{Morphological examination}

The morphological alteration was visually monitored using a Cytation 5 Cell Imaging Multi-Mode Reader system (BioTek, USA). Differentiating cells were imaged every $24 \mathrm{~h}$ for consecutive 4 days in the absence or presence of CAY10566.

\section{Gene expression assay}

Total RNA extraction was carried out using an RNA extraction kit (TaKaRa, Japan) following the instructions provided by the manufacturer. The quantity and quality of extracted RNA were checked using a spectrophotometer (NanoDrop Technologies, USA) and running on $1.5 \%$ agarose gel, respectively. The complementary DNA (cDNA) required for real-time PCR was synthesized with a cDNA synthesis kit (Roche, UK). Real-time PCR was performed with SYBR Green PCR master mix (Yekta Tajhiz Azma, Iran) on a MIC real-time PCR system (BioMolecular Systems, Australia). The primer sequences used for Sox2, Oct4, Sox17, and CXCR4 expression assay are listed in Additional file 1: Table S1. Relative gene expression was normalized against the expression level of GAPDH as a reference gene for each sample and all alterations were expressed as fold-changes relative to the mock.

\section{Surface markers analysis}

The pellet of disassociated cells was resuspended in $4 \%$ paraformaldehyde to fix the cells by a 30 min incubation at $4{ }^{\circ} \mathrm{C}$. Cells were then resuspended in PBS containing $1 \%$ BSA as a staining buffer and incubated for $20 \mathrm{~min}$ at $4{ }^{\circ} \mathrm{C}$. Then, cells were incubated with Alexa Fluor 488-conjugated CXCR4 (R\&D, USA) and PE-conjugated KDR (R\&D, USA), Alexa Fluor 488-conjugated SSEA-3, (eBioscience, USA), or PE-conjugated NCAM antibodies (BioLegend, USA) at $4{ }^{\circ} \mathrm{C}$ for $1 \mathrm{~h}$. After PBS washes, cells were analyzed using a flow cytometer (Miltenyi Biotec, USA) and the data were analyzed by the Flowing Software 2.5.1 (Turku Bioscience, Finland).

\section{Western blotting}

The cell lysate from each group was prepared using a RIPA lysis buffer containing protease inhibitor. Samples were centrifuged and the total protein concentration of supernatants was determined by Lowry protein assay. Twenty $\mu$ g of protein was mixed with an equal volume of sample buffer and electrophoresed on SDS-PAGE, then transferred onto PVDF membranes (Santa Cruz, USA). After blocking with 5\% skimmed milk, the membrane was washed in PBS with Tween detergent and treated with primary antibodies against the pluripotency markers Oct4 (Abcam, USA) and Sox2 (Abcam, UK), endodermic markers CXCR4 (Santa Cruz, USA), and Sox17 (Santa Cruz, USA), and the internal control $\beta$-actin (Santa Cruz Biotechnology, USA) overnight at $4{ }^{\circ} \mathrm{C}$. Primary antibodies against Wnt3a (Santa Cruz, USA) and $\beta$-catenin (Santa Cruz, USA) were applied for evaluation of the Wnt signaling pathway. Then, the membrane was exposed to horseradish peroxidase (HRP)-conjugated secondary antibody for $1 \mathrm{~h}$ at RT. Finally, the membrane was visualized by luminol reagents (Santa Cruz, USA). The intensity of the bands was quantified using ImageJ software (version 1.41). 


\section{Cell proliferation assay}

The proliferation rate was measured using a 5-bromo2-deoxyuridine (BrdU) assay (Abcam, USA). To this end, cells were seeded at a density of $2 \times 10^{4}$ cells per well of 96-well plates. After completion of the treatment protocol, each well was filled with BrdU reagent before cell harvesting. Cellular DNA was denatured with the fixing solution at RT. After washing with PBS, the anti-BrdU monoclonal antibody was added to each well, and plate was incubated for $1 \mathrm{~h}$ at RT. Then, supernatants were discarded and the washing step was repeated followed by the addition of an HRP-conjugated anti-IgG antibody. After the final wash, each well was incubated with a peroxidase substrate and then the reaction was stopped by adding the stop solution when the yellow color was generated. The optical density of the solution was measured using a microplate reader (BDSL Immunoskan, Finland) at $450 \mathrm{~nm}$. Wells containing media alone and seeded cells without BrdU reagent were used as blank and background controls, respectively.

\section{Acylation assay}

To evaluate the total protein acylation, we performed an alkyne-azide cycloaddition click reaction according to a standard protocol [27]. Briefly, cells were seeded onto a 24 -well plate at a density of $5 \times 10^{4}$ cell/well and treated as above-mentioned. Medium containing the $\omega$-alkynyl analog of palmitic acid (Alk-C16) was added into the wells at each time point after washing once with PBS and incubated for $24 \mathrm{~h}$ at $37{ }^{\circ} \mathrm{C}$ with $5 \% \mathrm{CO}_{2}$ to label the cells. After discarding the medium, cells were then washed with pre-cooled PBS, fixed with $-20{ }^{\circ} \mathrm{C}$ pre-chilled methanol. Permeabilization was performed using Triton X-100 at RT for 5 min. Cells were washed with PBS and exposed to a click labeling reagent containing Alexa Fluor 488 Azide (Invitrogen, USA), Tris(2-carboxyethyl) phosphine hydrochloride (TCEP) (Cayman, USA), and $\mathrm{CuSO}_{4}$ for $1 \mathrm{~h}$ at RT and dark. The wells were then washed with PBS, incubated with 4',6-diamidino-2-phenylindole (DAPI) for $30 \mathrm{~S}$ at RT, and washed three times with PBS. Finally, cells were imaged using the cell imaging system at excitation 488/emission $516 \mathrm{~nm}$ for Alexa Fluor488 and excitation $377 /$ emission $477 \mathrm{~nm}$ for DAPI.

\section{Cell cycle analysis}

Cell cycle assay was performed using a cell cycle phase determination kit (Cayman Chemical, USA). Cells were seeded on the culture plates according to the manufacturers' instructions. After completion of treatment protocols, the cells were trypsinized, centrifuged, and washed with the assay buffer. The cell pellet was resuspended in the assay buffer and incubated with a fixation buffer. Then, the cell suspension was centrifuged and the supernatant was discarded followed by incubation in staining solution at RT and dark. The percentage of cells in each phase was determined using Flowing software 2.5.1 (Turku Centre for Biotechnology, Finland).

\section{Statistical analysis}

Experimental data are presented as mean \pm standard deviation (SD) of the mean from at least three independent experiments. One-way or two-way analysis of variance (ANOVA) followed by Tukey's post hoc test was applied for groupwise comparisons (GraphPad Software 8.0, USA). A $p$ value less than 0.05 was considered to be significant.

\section{Results}

\section{SCD1 inhibition did not affect cell viability} and proliferation

In order to evaluate the toxic effect of inhibitor concentration on stem cell viability and proliferation, the hiPSCs and hESCs were treated with the SCD1 inhibitor at Day 0 of endoderm differentiation. The flow cytometry results demonstrated that the applied concentration of the inhibitor did not induce an apoptotic or necrotic effect on cells and the majority of cells were annexin- $\mathrm{V}$ and PI negative (Fig. 1). Similarly, the cell proliferation rate, when compared to the mock group, was not altered after inhibition of SCD1 at Days 0, 1, or 2 of endoderm differentiation $(p>0.05)$. According to our data, the proliferation rate was high; more than $70 \%$ of cells were BrdU positive at Day 4 of differentiation (Fig. 2).

\section{SCD1 inhibition maintained stem cell features}

Bright-field microscopic imaging displayed prominent morphological changes during endoderm induction (Additional file 2: Fig. S1). One day after the differentiation protocol, the cells lost their typical morphological characteristics (i.e., round and compact shapes with high nuclear/cytoplasm ratios) and morphologically different cells appeared. In the next days, the number of morphologically different cells increased. On Day 4, a monolayer of morphologically uniform cells with petal/cobblestonelike morphologies and optically clear cytoplasms were obtained (Additional file 2: Fig. S1). We showed that inhibition of SCD1 only at Day 0 did not change morphology stem cell. It is worth noting that the co-treatment of cells with oleate can change morphological features as appeared with endodermal induction (Fig. 3).

\section{SCD1 inhibition reduced the expression of endodermal markers}

In differentiated cells, the expression rates of the endodermal markers Sox17 and CXCR4 were robustly elevated, which coincided with the down-regulation of 



Fig. 1 Flow cytometric analysis of pluripotent stem cells after treatment with the SCD1 inhibitor. A Representative flow cytometry dot plots showing four-cell fractions: viable cells (annexin $\mathrm{V}^{-} / \mathrm{PI}^{-}$), early apoptotic cells (annexin $\mathrm{V}^{+} / \mathrm{PI}^{-}$), late apoptotic cells (annexin $\mathrm{V}^{+} / \mathrm{Pl}^{+}$), and necrotic cells (annexin $\mathrm{V}^{-} /\left.\mathrm{PI}\right|^{+}$). The induced pluripotent stem cells (iPSCs) and embryonic stem cells (ESCs) were mock-treated with DMSO $(<0.05 \%$, above) or treated with $25 \mathrm{nmol} / \mathrm{L}$ of SCD1 inhibitor (SCDinhib) on Day 0 of differentiation (below) for 4 days. Cells were then harvested and evaluated for apoptosis and necrosis. B Quantification of cells in different groups



Fig. 2 Cell growth determination using BrdU incorporation assay. The induced pluripotent stem cells (iPSCs) and embryonic stem cells (ESCS) were treated with SCD1 inhibitor (SCDinhib) alone at Days 0, 1 , or $2(0,1$, and 2$)$ of differentiation or in combination with oleic acid (OA) on Day 0. Undifferentiated cells (Undiff) and cells treated with DMSO $(<0.05 \%$, Mock) were served as background controls. The cells were harvested on Day 4 pluripotency markers Oct4 and Sox2 (Fig. 4). These findings indicate the efficiency of the current protocol to commit the cells toward an endoderm-like cell population. To examine the effect of SCD1 inhibition on differentiation of hiPSCs toward endodermal lineage, the one-shot treatment of cells with a non-toxic concentration of SCD1 inhibitor was performed on Day 0, Day 1, and Day 2 of the differentiation process. The alterations of pluripotency and endodermal markers were assessed at both gene and protein levels. When compared to the mock condition, it could be seen that the inhibition of SCD1 significantly prevented the down-regulation of pluripotency marker Oct4 and Sox 2 and the increase in endodermal lineage gene markers Sox 17 and CXCR4 (Fig. 4). Based on our data, the effect of SCD1 inhibition was smaller on Day 2 of differentiation. As indicated in Fig. 4, in undifferentiated hiPSCs, the expressions of the stemness marker Oct 4 were 21.39 -fold (gene, $p<0.01$ ) and 3.67 -fold (protein, $p<0.01$ ) whereas these values were $16.01(p<0.01)$ and $3.2(p<0.01)$ for Sox2. According to our data, both the transcription and protein levels 


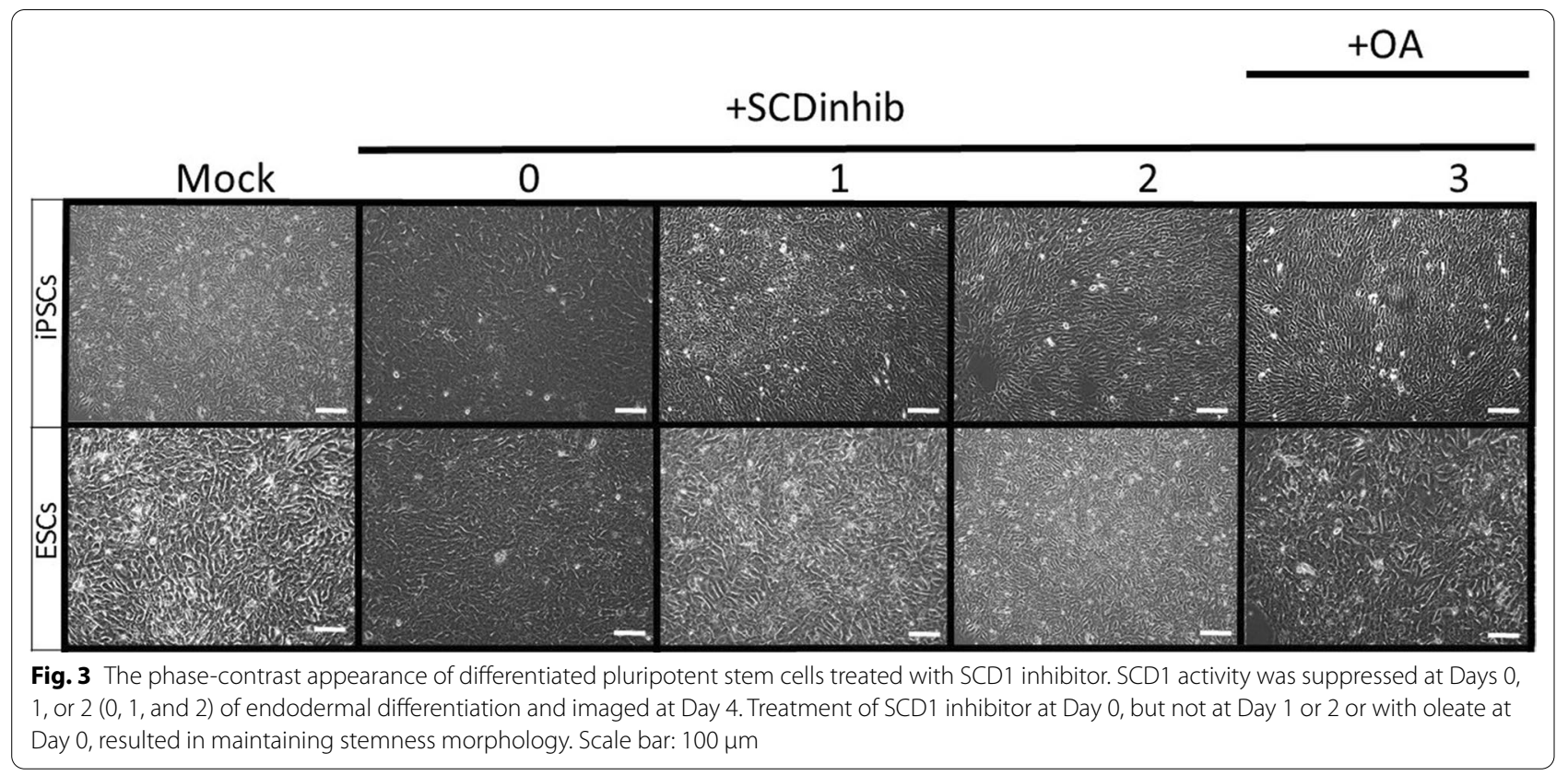

of CXCR4 and Sox17 reached 0.04-fold (gene, $p<0.01$ ), 0.22 -fold (protein, $p<0.01$ ), 0.04 -fold (gene, $p<0.01$ ), and 0.17 -fold $(p<0.01)$ compared to the mock condition.

In differentiated cells, the transcription and protein levels of Oct 4 were 13.02-fold (gene, $p<0.01$ ) and 2.88fold (protein, $p<0.01$ ) higher than those of the mock, respectively. Notably, when SCD1 was inhibited on Day 0 , the values for Sox 2 were 9.63-fold (gene, $p<0.01$ ) and 2.76 -fold (protein, $p<0.01$ ) higher compared to the mock condition. We noted that the expressions of stemness markers were not significantly altered when SCD1 was inhibited on Day 1, except for Sox2, which was 6.2-fold (gene, $p<0.05$ ) higher in comparison to the mock. In differentiated cells, the expressions of the endoderm marker CXCR4 were 0.39 -fold (gene, $p<0.01$ ) and 0.27 -fold (protein, $p<0.01$ ) lower. The expressions of Sox 17 were 0.27 fold (gene, $p<0.01$ ) and 0.24-fold (protein, $p<0.01$ ) lower compared to the mock group when SCD1 was inhibited on Day 0 (Fig. 4). The assessment of endodermal markers revealed that with Day 1 inhibitor the transcription of Sox17 was 0.43 -fold (gene, $p<0.01$ ) lower than the mock group. The ESCs demonstrated a similar pattern of results after the inhibition of SCD1 during endodermal inhibition. As expected, in the rescue experiment, using oleate together with the SCD1 inhibitor, the endoderm markers efficiently recovered (Fig. 4).

\section{SCD1 inhibition did not divert differentiation of hiPSCs toward mesodermal or ectodermal lineages}

A combination of surface markers, representing the three germ layers cells and undifferentiated cells, was analyzed to distinguish cell types upon SCD1 inhibition and endodermal differentiation. We found that the percentage of endoderm cells decreased 2.56-fold $(p<0.01)$ with the inhibition of SCD1. Interestingly, the percentage of undifferentiated cells in the mock group increased 8.2fold $(p<0.01)$ and 2.49 -fold $(p<0.05)$ following the inhibition of SCD1 on Days 0 and 1, respectively. With the addition of oleate together with SCD1 inhibitor on Day 0 , the percentage of endoderm cells increased 2.4-fold $(p<0.01)$ and undifferentiated cells decreased to 6.53-fold $(p<0.01)$, respectively, compared to the same day only with the SCD1 inhibitor. Notably, the inhibition of SCD1 did not significantly alter the percentages of the ectodermal or mesodermal population (Fig. 5).

\section{SCD1 inhibition resulted in a decrease in total protein acylation}

To evaluate the effect of SCD1 inhibition on the acylation of total proteins during endoderm induction, the total protein acylation rate of pluripotent stem cells was measured using click chemistry. Fluorescent imaging results demonstrated a high and constant acylation rate during normal endoderm differentiation (Additional file 3: Fig. S2). However, the inhibition of SCD1 activity at Days 0, 1 , or 2 decreased the intensity of the fluorescent emission, which was significant on Day 0. In particular, after the inhibition of SCD1 at Day 0, the emission intensity reached 0.59 -fold $(p<0.05)$ and 0.67 -fold $(p<0.05)$ lower in the hiPSCs (Fig. 6A, B) and ESCs (Fig. 6C, D), respectively, compared to the mock condition. A decrease in the fluorescent emission was also observed on Day 1 by the 

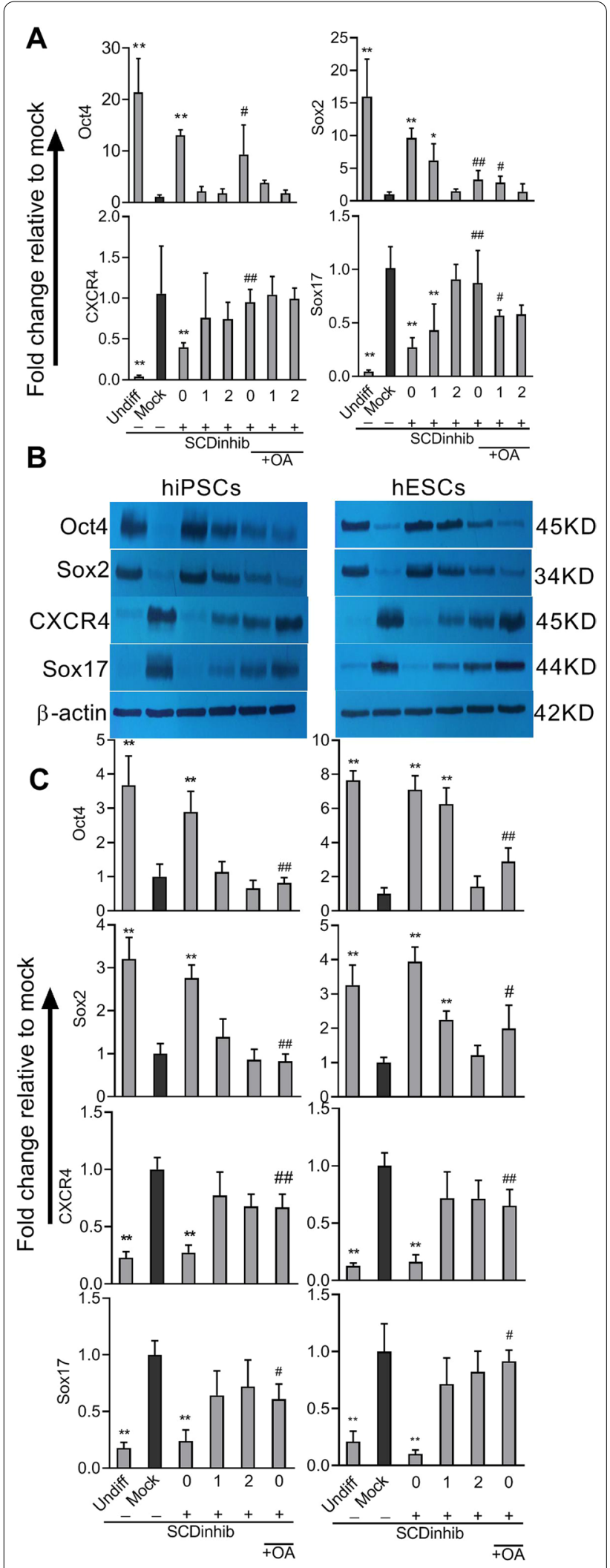

Fig. 4 The expression of endoderm and stemness markers following the inhibition of SCD1 activity during induced differentiation. Cells were treated with SCD1 inhibitor (SCDinhib) alone at Days 0, 1, or 2 $(0,1$, and 2$)$ of differentiation or in combination with oleic acid (OA) at Day 0. Undifferentiated cells (Undiff) and cells treated with DMSO $(<0.05 \%$, Mock) were served as background controls. The cells were harvested on Day 4. The gene expression in iPSCs (A) and protein expression (representative Western blot and quantification) (B) of the stemness markers Oct4 and Sox2 and endoderm markers CXCR4 and Sox17 were evaluated in the induced pluripotent stem cells (iPSCS) and embryonic stem cells (ESCs) using quantitative PCR and Western blot, respectively. $\mathbf{C}$ Quantification of protein expression in different groups. ${ }^{*} p<0.05,{ }^{* *} p<0.01$ versus mock, ${ }^{\#} p<0.05$, ${ }^{\#} p<0.01$ versus the same day in SCDinhib

inhibition of SCD1 in iPSCs $(0.63$-fold of mock, $p<0.05$, Fig. 6A, B).

\section{SCD1 inhibition increased the percentage of cells in the $S$ phase}

The distributions of differentiated hiPSCs in the cell cycle phases were determined using single color flow cytometry analysis after DNA staining with PI. In the undifferentiated state, the percentages of cells in the G1 and S phases were 19.14 and 56.04, respectively, showing characteristics of PSCs (Fig. 7). In this condition, the percentages of cells in $\mathrm{G} 1$ and $\mathrm{S}$ phases were 0.53 -fold $(p<0.01)$ and 1.5 -fold $(p<0.05)$ of the mock group, respectively. The inhibition of SCD1 activity reduced the percentage of cells in the G1 phase $(0.58$-fold, $p<0.05)$ on Day 0 and concomitantly increased the percentage of cells in the $\mathrm{S}$ phase $(1.52$-fold, $p<0.05)$ as compared to the mock group. In the rescue experiment, the percentages of cells in the $\mathrm{G} 1$ and $\mathrm{S}$ phases were close to the mock condition. The percentages of cells in the G1 phase increased 1.74 and in the $\mathrm{S}$ phases decreased 0.69 -fold, respectively, compared to time-matched groups that received SCD1 inhibitor (Fig. 7).

\section{Inhibition of SCD1 decreased endoderm differentiation through attenuation of the Wnt signaling pathway}

Western blotting showed that the protein levels of Wnt3a and its downstream $\beta$-catenin gradually decreased from Day 0 to Day 4 of endoderm differentiation (Additional file 4: Fig. S3). The data show a lack of significant difference in the expression of $\beta$-catenin following the inhibition of SCD1 on Days 0,1 , or 2 of differentiation. Upon SCD1 inhibition on Day 0 in iPSCs and ESCs, the expression of Wnt3a was 1.57- and 1.26-fold higher than the values observed in the same days without the inhibition of SCD1 $(p<0.05)$, respectively. As expected, the addition of oleate at the same time efficiently counteracted 


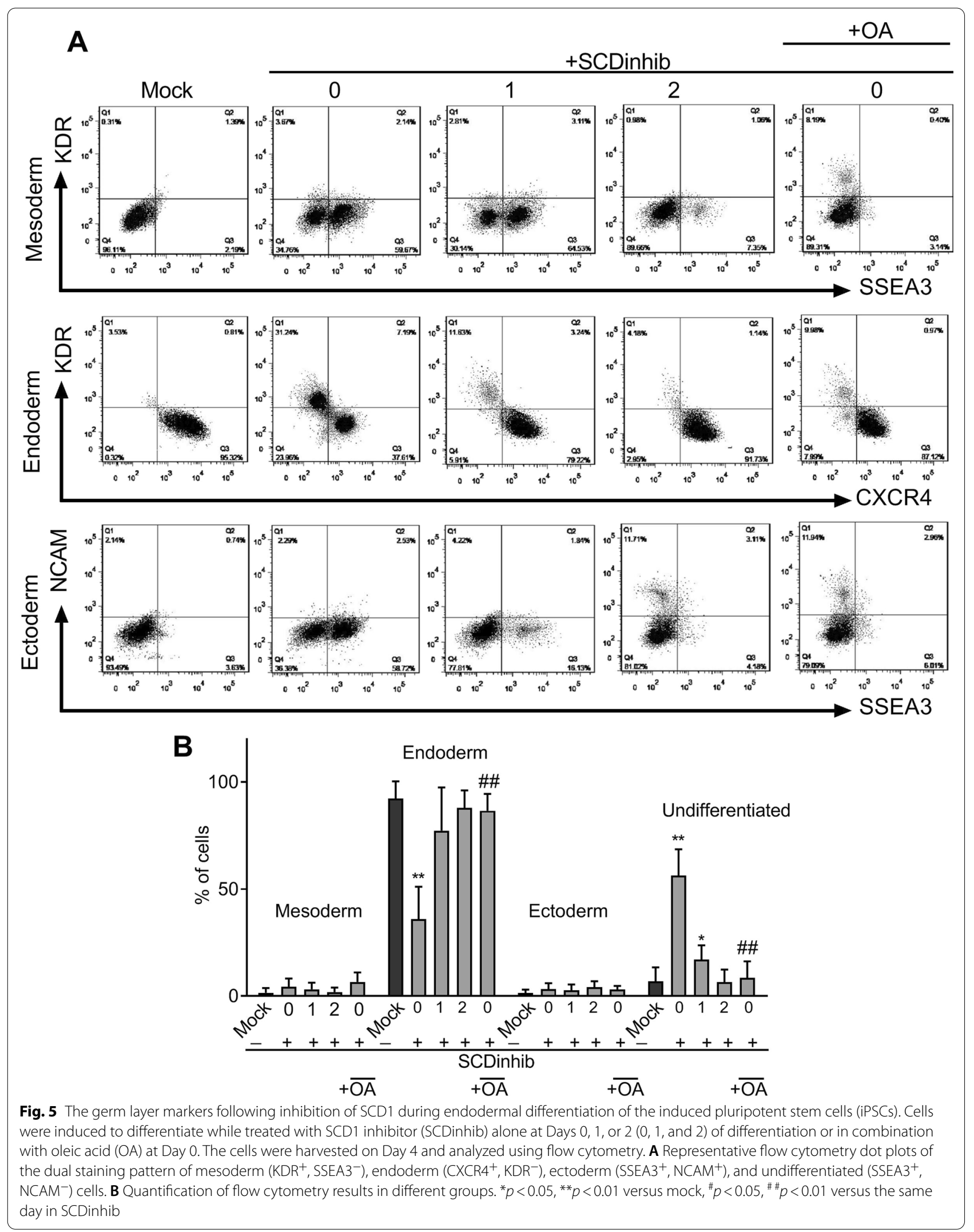






the increasing and decreasing effects of the inhibitor on Wnt3a and $\beta$-catenin, respectively (Fig. 8).

\section{Discussion}

PSCs with a high potential to differentiate into adult functional cells are promising options for the treatment of human degenerative diseases [28]. The application of these cells in the clinical setting is mainly hindered by the low differentiation efficiency of in vitro protocols and the possibility of tumorigenicity after transplantation into target organs $[29,30]$. In addition, the self-differentiation of these cells during expansion makes the handling of these cells more difficult. The subtle and precise control of differentiation is critical to overcoming these challenges. By increasing our knowledge of the molecular mechanisms governing cell differentiation, we can find an optimum condition for differentiation and maintenance of stem cell behavior in preclinical models or in clinical applications.

MUFAs play important roles in developmental processes through post-translational modification of the proteins involved in differentiation. Thus, understanding the 


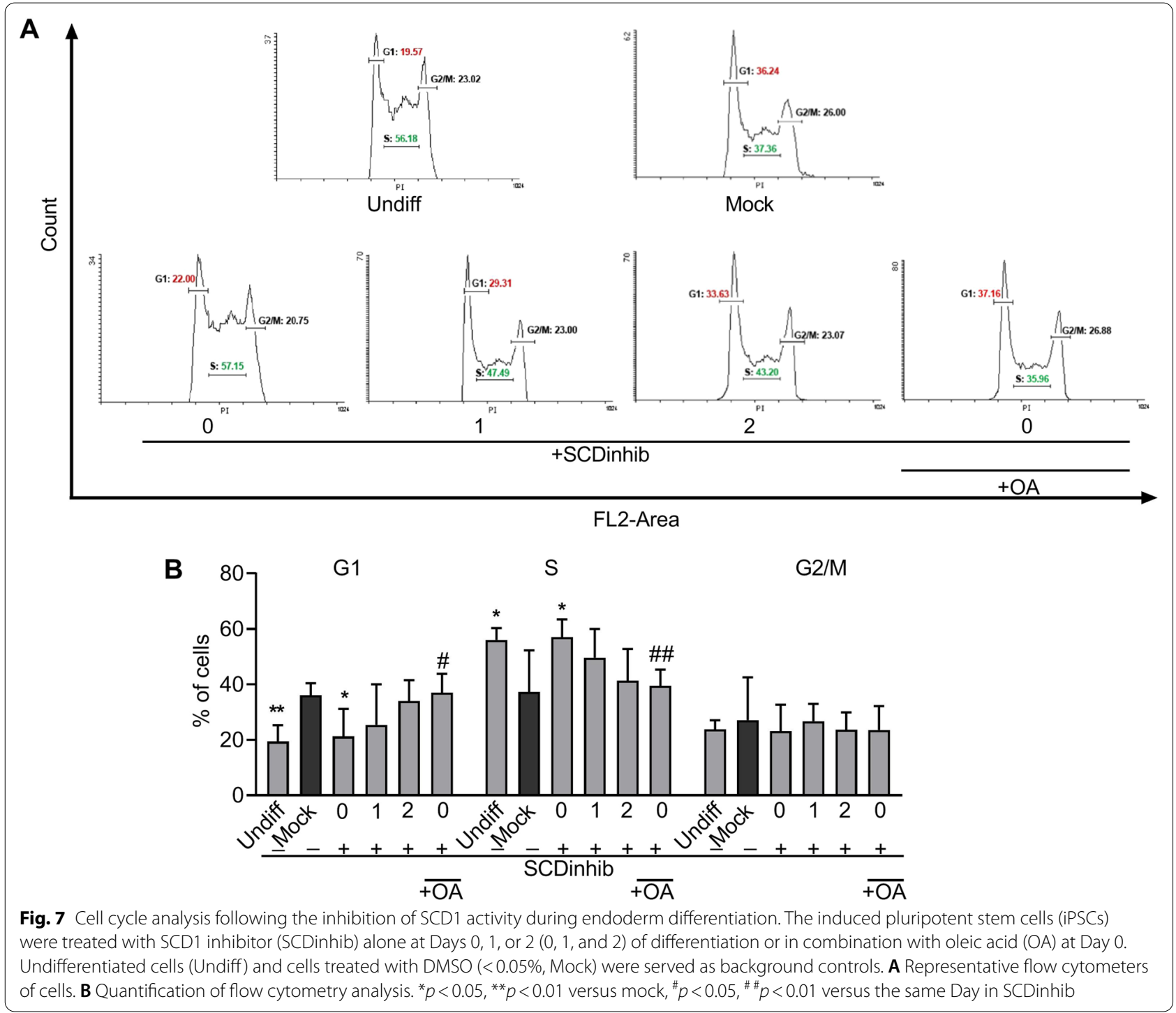

mechanisms by which the metabolism of MUFAs affects stem cell behavior offers promising perspectives to help regulate stem cell differentiation pharmacologically. In the current study, we evaluated the effect of SCD1 inhibition on endodermal differentiation in PSCs by monitoring their protein and transcription levels of molecular markers. We also monitored its effect on total protein acylation, cell viability, cell proliferation, cell cycle, and the Wnt signaling pathway. To assess the specificity of the effects, the main product of SCD1 oleate was co-administrated with the chemical inhibitor.

We observed no significant changes in the PSC proliferation, apoptosis, and necrosis rates after SCD1 inhibition compared to the control group. Therefore, the effects of SCD1 inhibition on PSCs are seemingly independent from cell proliferation, apoptosis, or necrosis. Consistent with our data, the inhibition of SCD1 with similar concentrations of inhibitor did not alter cell viability during liver cell differentiation of hiPSCs [22]. However, treatments of ESC-derived endoderm progenitor cells, ESC-derived hepatocytes, and iPSCderived cardiomyocytes with the toxic concentration of SCD1 inhibitor were shown to selectively eliminate the cells in undifferentiated states [17]. Notably, the incubation of lung cancer cells with the SCD1 inhibitor-induced toxicity in cancer stem cells [31]. These findings report that the inhibition of SCD1 in different progenitor lineages can yield different outcomes in dynamic growth. The exact mechanisms and underlying machinery participating in this phenomenon are unknown. Our findings showed that the inhibition of SCD1 can reduce the PSC differentiation capacity toward endodermal lineage while maintaining pluripotency of the cells. It confirmed that the efficiency 

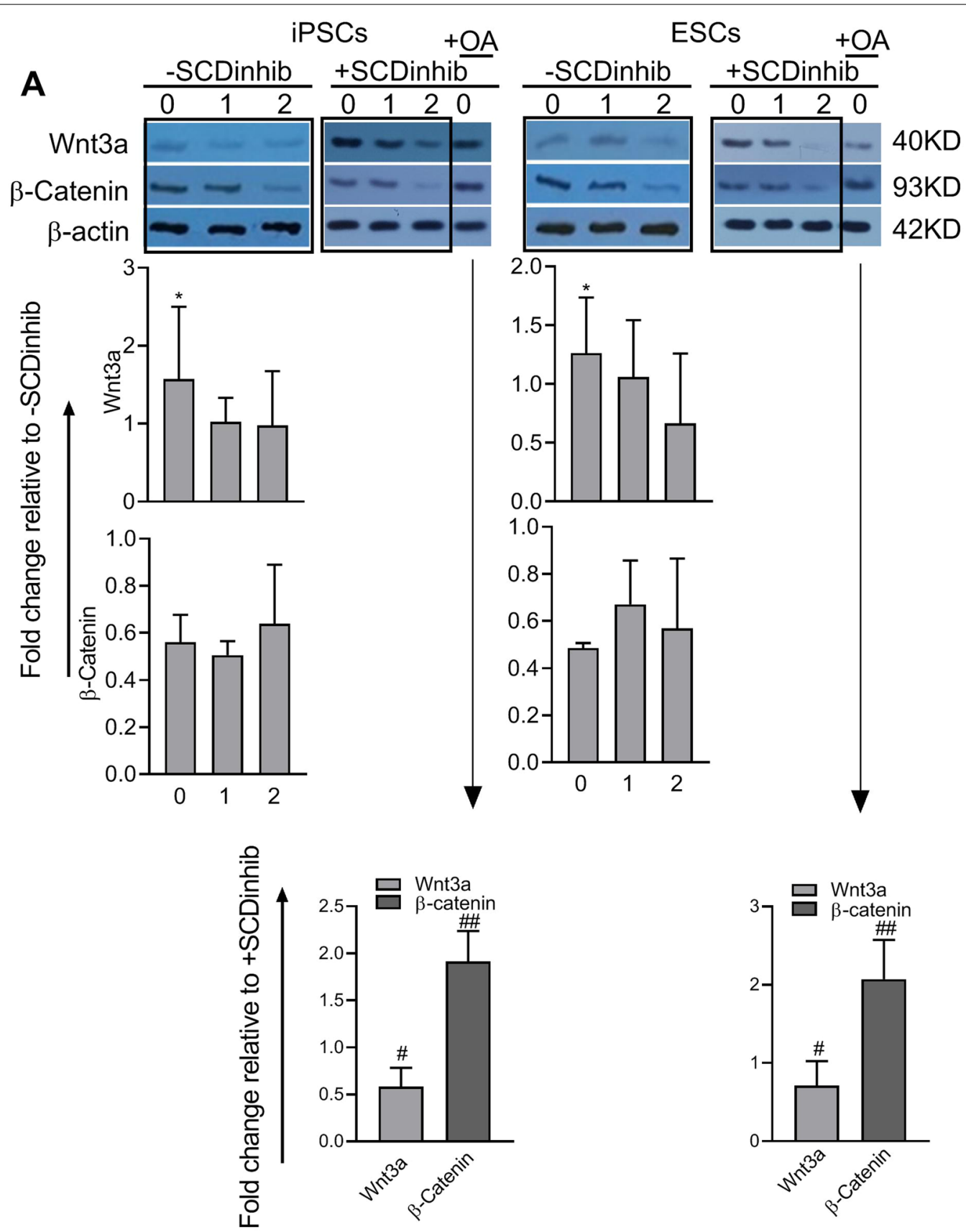

Fig. 8 Analysis of the Wnt signaling pathway following endoderm differentiation of pluripotent stem cells. The induced pluripotent stem cells (iPSCs) and embryonic stem cells (ESCS) were treated with SCD1 inhibitor (SCDinhib) alone at Days 0, 1, or $2(0,1$, and 2) of differentiation or in combination with oleic acid (OA) at Day 0 . A The representative Western blot and the quantification of Wnt $3 a$ and $\beta$-catenin. ${ }^{*} p<0.05$ versus mock, ${ }^{\#} p<0.05,{ }^{\#} p<0.01$ versus the same Day in SCDinhib

of SCD1 inhibition varied with time during endoderm differentiation. Following the inhibition SCD1 on Day 0 of differentiation, the synthesis of endoderm markers was markedly suppressed. As expected, the treatment of the cells with the SCD1 inhibitor together with exogenous oleate recovered the cells from these effects. The fundamental cellular mechanism that supports these effects is that palmitoleate, like oleate, is also the product of SCD1. It seems that oleate alone can compensate for the deficiency of SCD1 activity during endodermal differentiation of PSCs. In line with our study, the suppression of SCD1 activity during hepatic differentiation 
delayed the production of hepatic function markers during liver cell maturation [22]. Presumably, the activity of SCD1 is reduced concomitantly by the progression of cells toward specialized cells [22]. Data further confirmed the pivotal role of SCD1 in the development of the liver in rat embryos [23]. The inhibition of SCD1 in an early phase of pregnancy led to severe defects in fetal liver development indicated by the down-regulation of HNF1 $\alpha$, AFP, ALB, and CYP450 [23]. These effects were blunted in the presence of oleate [22, 23]. It was suggested that using IWP-2 to inhibit Porcn, an acyltransferase catalyzing the addition of MUFAs onto Wnts, reduced the expression of endoderm markers such as Sox17 and FOXa2 a few hours before initiation of endoderm induction [32]. Along with our results, it can be proposed that SCD1 and its products are involved in the development and differentiation of stem cells into the endodermal lineages [33, 34]. In contrast, some reports demonstrated different outcomes associated with the function of SCD1 products in stem cells. It was recently found that the treatment of MEFs during their reprogramming into iPSCs resulted in iPSCs that formed more colonies in comparison to the nontreated control. Furthermore, these iPSCs had a higher capacity for palmitoleate and oleate synthesis. Remarkably, the inhibition of Porcn can lead to a reduction in the pluripotency of the ESCs [35]. These results indicate that SCD1 products can act in a context-dependent manner in terms of cell fate acquisition. The function of SCD1 products can promote cell differentiation and are critical in the early phase of differentiation. Meanwhile, they can help to maintain stem cell features. Similar effects have been reported in molecules dependent on MUFAs, such as Wnt molecules. Several conflicting reports in the literature show that Wnt molecules promote either stemness or differentiation and the lineage specification of stem cells [36-40].

To investigate the possible role of SCD1 in the proteins acylation rate, we used click chemistry and fluorescent imaging during endoderm differentiation and following the SCD1 inhibition. An Alk-C16 probe analysis showed that endoderm differentiation is associated with a significant protein acylation signal. This finding implies that a great deal of proteins undergo acylation by fatty acids in stem cells subjected to endodermal differentiation. Previously, it has been shown that AlkC16 can be converted into its monounsaturated form Alk-C16:1 by the activity of SCD1 [41]. Therefore, the intensity of the signal is associated with the incorporation of both saturated and monounsaturated forms of the probe. Notably, the acylation rate significantly decreased when SCD1 was inhibited on Day 0. We interpreted this event as correlating with the reduction of the monounsaturated form of Alk-C16 for incorporation and biochemical reactions.

Previous studies showed the significant role of Activin $A$ and $W n t / \beta$-catenin pathways in the endodermal differentiation of ESCs [32, 42, 43]. Activin A-induced SCD1 can produce metabolites that can post-translationally modify the Wnt molecules [43]. The contribution of SCD1 in the activity of Wnt signaling was assessed using western blotting and gene expression analysis. Our results showed mild to moderate suppression of Wnt3a/ $\beta$-catenin during the differentiation of iPSCs toward endodermal lineages, indicating the crucial role of SCD1 in early-stage stem cell differentiation. Following the treatment of cells with the SCD1 inhibitor, the expression of Wnt was increased with non-significant changes in $\beta$-catenin. One reason for the Wnt induction may be that its expression is a compensatory response after the suppression of MUFA acylation via the SCD1 product. Additionally, the slight-to-mild suppression of $\beta$-catenin can be related to the attenuation of the Wnt signaling pathway following SCD1 inhibition, as these features result in the reduced endoderm differentiation of iPSCs. Based on these results, we hypothesize that the changes in the Wnt $/ \beta$-catenin signaling pathway are possibly due to reduced MUFA acylation. Moreover, it has been noted that SCD1 activity can be changed during cell differentiation. Evaluation of other MUFAs with respect to different types of Wnt molecules such as Wnt5a will enable us to understand the role of MUFA acylation in diverse differentiation process. MUFAs are much more essential in the first stages of differentiation than in the latter steps, indicating that the immediate triggering activity of SCD1 is essential for endodermal differentiation in hiPSCs. As indicated previously, the generation of the primitive streak and subsequently formation of mesendodermal cells are a prerequisite for the formation of endoderm $[26,44]$. Most probably, MUFAs are required for the production of primitive streak- and mesendoderm-like cells; however, the hypothesis needs to be investigated in future studies.

\section{Conclusion}

The dynamics of SCD1 activity are crucial to the early commitment and differentiation of hiPSCs to endoderm lineage. We showed that SCD1 inhibition attenuates the Wnt/ $\beta$-catenin signaling pathway, conferring the maintenance of hiPSCs by opposing the initiation of endoderm differentiation. The requirement for SCD1 activity in the endoderm commitment of PSCs may be of importance in disorders of endoderm-derived organs and dysregulated metabolism. 


\section{Abbreviations}

Alk-C16: $\omega$-Alkynyl analog of palmitic acid; b-FGF: Basic human recombinant FGF; BrdU: 5-Bromo-2-deoxyuridine; D-FBS: Defined-FBS; FBS: Fetal bovine serum; hESCs: Human embryonic stem cells; hiPSCs: Human-induced pluripotent stem cells; miPSCs: Mouse-induced pluripotent stem cells; MUFAs: Monounsaturated fatty acids; PBS: Phosphate-buffered saline; SCD1: Stearoylcoenzyme A desaturase 1; SD: Standard deviation; SFAs: Saturated fatty acids; TCEP: Tris(2-carboxyethyl) phosphine hydrochloride.

\section{Supplementary Information}

The online version contains supplementary material available at https://doi. org/10.1186/s13287-021-02617-x.

Additional file 1: Table S1. List of primers used for gene expression analysis in this study.

Additional file 2: Fig. S1. The phase-contrast appearance of differentiating pluripotent stem cells. The induced pluripotent stem cells (iPSCs) and embryonic stem cells (ESCs) were differentiated toward endoderm lineage with activin A and defined FBS. Cells exhibited typical morphology of pluripotent stem cells on Day 0. Over differentiation, a loss of typical stem cell morphology was noted on Day 2. Very different cellular morphologies appeared in the following days until at Day 4 a monolayer of morphologically uniform cells was obtained. Scale bar: $100 \mu \mathrm{m}$.

Additional file 3: Fig. S2. Click chemistry for imaging of whole protein acylation during endoderm differentiation of the induced pluripotent stem cells (iPSCs). Cells were induced to differentiate and imaged with $24 \mathrm{~h}$ intervals of 0 to 4 days $(0,1,2,3$, and 4$)$. A Cells were treated with Alk-C16 before reaction with Alexa Fluor 488 azide (green). Nuclei were stained with DAPI (blue) to normalize for the cell number. B Quantification of fluorescence density. Alk-C16; $\omega$-alkyne palmitic acid. Scale bar: $100 \mu \mathrm{m}$.

Additional file 4: Fig. S3. Analysis of the Wnt signaling pathway during endoderm differentiation of pluripotent stem cells. The induced pluripotent stem cells (iPSCs) and embryonic stem cells (ESCs) were induced to differentiate and analyzed at Days $0,1,2,3$, and $4(0,1,2,3$, and 4). The cells were harvested on Day 4 . A The representative Western blot of Wnt3a and $\beta$-catenin. B Quantification of protein expression in different groups. ${ }^{*} p<0.05$ and ${ }^{* *} p<0.01$ versus Day $0,{ }^{\#} p<0.05,{ }^{\# \#} p<0.01$ versus Day 1 , $\$ p<0.05, \$ \$ p<0.01$ versus Day $2,{ }^{\circledR} p<0.05,{ }^{@} p<0.01$ versus Day 3.

\section{Acknowledgements}

Not applicable.

\section{Authors' contributions}

MD conceived and designed the experiments. VH performed cell isolation and molecular analyses and wrote the manuscript draft. $\mathrm{VH}$ and $\mathrm{MH}$ performed cell culture. AKC and PF performed extractions and blotting. MD, MT, RR, and AKC reviewed the manuscript. All authors read and approved the final manuscript.

\section{Funding}

The research protocol was approved and supported by the Student Research Committee (59924), Stem Cell Research Center at Tabriz University of Medical Sciences (67634), and the National Council for Development of Stem Cell Sciences and Technologies (394).

\section{Availability of data and materials}

Not applicable.

\section{Declarations}

\section{Ethics approval and consent to participate}

The study protocols were approved by the Ethics Committee of Tabriz University of Medical Sciences (IR.TBZMED.REC.1395.680). All animals were treated according to guidelines for the Care and Use of Laboratory Animals (NIH; 1986).

\section{Consent for publication}

Not applicable.

\section{Competing interests}

The authors declare that they have no competing interests.

\section{Author details}

${ }^{1}$ Student Research Committee, Tabriz University of Medical Sciences, 5166615573 Tabriz, Iran. ${ }^{2}$ Stem Cell Research Center, Tabriz University of Medical Sciences, 516615731 Tabriz, Iran. ${ }^{3}$ Department of Biochemistry and Clinical Laboratories, Faculty of Medicine, Tabriz University of Medical Sciences, Tabriz, Iran. ${ }^{4}$ Department of Clinical Biochemistry, Faculty of Medicine, Hamadan University of Medical Sciences, Hamadan, Iran. ${ }^{5}$ Department of Stem Cells and Developmental Biology, Cell Science Research Center, Royan Institute for Stem Cell Biology and Technology, ACECR, Tehran, Iran. ${ }^{6}$ Department of Applied Cell Sciences, Faculty of Advanced Medical Sciences, Tabriz University of Medical Sciences, Tabriz, Iran. ${ }^{7}$ Department of Genetics, Reproductive Biomedicine Research Center, Royan Institute for Reproductive Biomedicine, ACECR, Tehran, Iran. ${ }^{8}$ Department of Internal Medicine IV, Heidelberg University Hospital, Heidelberg, Germany.

Received: 1 August 2021 Accepted: 7 October 2021

Published online: 21 October 2021

\section{References}

1. Yu J, Vodyanik MA, Smuga-Otto K, Antosiewicz-Bourget J, Frane JL, Tian S, Nie J, Jonsdottir GA, Ruotti V, Stewart R. Induced pluripotent stem cell lines derived from human somatic cells. Science. 2007;318(5858):1917-20.

2. Yamanaka S. Induced pluripotent stem cells: past, present, and future. Cell Stem Cell. 2012;10(6):678-84.

3. Liu G, David BT, Trawczynski M, Fessler RG. Advances in pluripotent stem cells: history, mechanisms, technologies, and applications. Stem Cell Rev Rep. 2020;16(1):3-32.

4. Rowe RG, Daley GQ. Induced pluripotent stem cells in disease modelling and drug discovery. Nat Rev Genet. 2019;20(7):377-88.

5. Hirabayashi M, Goto T, Hochi S. Pluripotent stem cell-derived organogenesis in the rat model system. Transgenic Res. 2019;28:1-11.

6. Hosseini V, Maroufi NF, Saghati S, Asadi N, Darabi M, Ahmad SNS, Hosseinkhani H, Rahbarghazi R. Current progress in hepatic tissue regeneration by tissue engineering. J Transl Med. 2019;17(1):383.

7. Nagy K, Tiuca I-D. Importance of fatty acids in physiopathology of human body. In: Catala A, editor. Fatty acids. IntechOpen; 2017.

8. Ntambi J. Dietary regulation of stearoyl-CoA desaturase 1 gene expression in mouse liver. J Biol Chem. 1992;267(15):10925-30.

9. Mohammadzadeh F, Hosseini V, Alihemmati A, Mehdizadeh A, Shaaker M, Mosayyebi G, Darabi M. The role of stearoyl-coenzyme a desaturase 1 in liver development, function, and pathogenesis. J Renal Hepatic Disord. 2019;3(1):15-22.

10. Kalantary-Charvadeh A, Hosseini V, Mehdizadeh A, Darabi M. Application of porcupine inhibitors in stem cell fate determination. Chem Biol Drug Des. 2020;96(4):1052-68.

11. Luis TC, Weerkamp F, Naber BA, Baert MR, de Haas EF, Nikolic T, Heuvelmans S, De Krijger RR, van Dongen JJ, Staal FJ. Wnt3a deficiency irreversibly impairs hematopoietic stem cell self-renewal and leads to defects in progenitor cell differentiation. Blood. 2009;113(3):546-54.

12. Lu W, Kim K-A, Liu J, Abo A, Feng X, Cao X, Li Y. R-spondin1 synergizes with Wnt3A in inducing osteoblast differentiation and osteoprotegerin expression. FEBS Lett. 2008;582(5):643-50.

13. Jia X, Wu B, Huang J, Fan L, Yang M, Xu W. YAP and Wnt3a independently promote AEClls proliferation and differentiation by increasing nuclear $\beta$-catenin expression in experimental bronchopulmonary dysplasia. Int J Mol Med. 2021;47(1):195-206.

14. Liang R, Xiao X, Luo L, Chen T, Yang H, Wang W, Zhang Y, Wang Z. Efficient definitive endoderm differentiation from human parthenogenetic embryonic stem cells induced by activin A and Wnt3a. Ann Clin Lab Sci. 2020;50(4):468-73.

15. Hay DC, Fletcher J, Payne C, Terrace JD, Gallagher RC, Snoeys J, Black JR, Wojtacha D, Samuel K, Hannoun Z. Highly efficient differentiation 
of hESCs to functional hepatic endoderm requires ActivinA and Wnt3a signaling. Proc Natl Acad Sci. 2008;105(34):12301-6.

16. Fathi Maroufi N, Hasegawa K, Vahedian V, Nazari Soltan Ahmad S, Zarebkohan A, Miresmaeili Mazrakhondi SA, Hosseini V, Rahbarghazi R. A glimpse into molecular mechanisms of embryonic stem cells pluripotency: current status and future perspective. J Cell Physiol. 2020;235:6377-92.

17. Ben-David U, Gan Q-F, Golan-Lev T, Arora P, Yanuka O, Oren YS, LeikinFrenkel A, Graf M, Garippa R, Boehringer M. Selective elimination of human pluripotent stem cells by an oleate synthesis inhibitor discovered in a high-throughput screen. Cell Stem Cell. 2013;12(2):167-79.

18. Zhang L, Pan Y, Qin G, Chen L, Chatterjee T, Weintraub N, Tang Y. Inhibition of stearoyl-coA desaturase selectively eliminates tumorigenic Nanog-positive cells: improving the safety of iPS cell transplantation to myocardium. Cell Cycle. 2014;13(5):762-71.

19. Janikiewicz J, Hanzelka K, Dziewulska A, Kozinski K, Dobrzyn P, Bernas T, Dobrzyn A. Inhibition of SCD1 impairs palmitate-derived autophagy at the step of autophagosome-lysosome fusion in pancreatic $\beta$-cells. J Lipid Res. 2015;56(10):1901-11.

20. Ntambi JM, Miyazaki M. Regulation of stearoyl-CoA desaturases and role in metabolism. Prog Lipid Res. 2004;43(2):91-104.

21. Ralston JC, Mutch DM. SCD1 inhibition during 3T3-L1 adipocyte differentiation remodels triacylglycerol, diacylglycerol and phospholipid fatty acid composition. Prostaglandins Leukot Essent Fatty Acids. 2015;98:29-37.

22. Rahimi Y, Mehdizadeh A, Nozad Charoudeh H, Nouri M, Valaei K, Fayezi S, Darabi M. Hepatocyte differentiation of human induced pluripotent stem cells is modulated by stearoyl-CoA desaturase 1 activity. Dev Growth Differ. 2015;57(9):667-74

23. Mohammadzadeh F, Alihemmati A, Tazehkand AP, Darabi M, Mehdizadeh A. Early oleate deficiency leads to severe defects in fetal rat liver development. Iran J Basic Med Sci. 2019;22(9):1010.

24. Hosseini V, Kalantary-Charvadeh A, Hasegawa K, Ahmad SNS, Rahbarghazi R, Mahdizadeh A, Darabi M, Totonchi M. A mechanical non-enzymatic method for isolation of mouse embryonic fibroblasts. Mol Biol Rep. 2020:47:1-10

25. Baharvand $H$, Ashtiani SK, Taee A, Massumi M, Valojerdi MR, Yazdi PE, Moradi SZ, Farrokhi A. Generation of new human embryonic stem cell lines with diploid and triploid karyotypes. Dev Growth Differ. 2006;48(2):117-28.

26. D'Amour KA, Agulnick AD, Eliazer S, Kelly OG, Kroon E, Baetge EE. Efficient differentiation of human embryonic stem cells to definitive endoderm. Nat Biotechnol. 2005;23(12):1534-41.

27. Gao X, Hannoush RN. Single-cell in situ imaging of palmitoylation in fatty-acylated proteins. Nat Protoc. 2014;9(11):2607.

28. Trounson A, DeWitt ND. Pluripotent stem cells progressing to the clinic. Nat Rev Mol Cell Biol. 2016;17(3):194-200.

29. Deng J, Zhang Y, Xie Y, Zhang L, Tang P. Cell transplantation for spinal cord injury: tumorigenicity of induced pluripotent stem cell-derived neural stem/progenitor cells. Stem Cells Int. 2018;2018:1-7.

30. Singh VK, Kalsan M, Kumar N, Saini A, Chandra R. Induced pluripotent stem cells: applications in regenerative medicine, disease modeling, and drug discovery. Front Cell Dev Biol. 2015;3:2.
31. Pisanu ME, Noto A, De Vitis C, Morrone S, Scognamiglio G, Botti G, Venuta F, Diso D, Jakopin Z, Padula F. Blockade of Stearoyl-CoA-desaturase 1 activity reverts resistance to cisplatin in lung cancer stem cells. Cancer Lett. 2017:406:93-104.

32. Xu H, Tsang KS, Wang Y, Chan JC, Xu G, Gao W-Q. Unfolded protein response is required for the definitive endodermal specification of mouse embryonic stem cells via Smad 2 and $\beta$-catenin signaling. J Biol Chem. 2014;289(38):26290-301.

33. Lee H, Lim J-Y, Choi S-J. Role of I-carnitine and oleate in myogenic differentiation: implications for myofiber regeneration. J Exerc Nutr Biochem. 2018;22(2):36.

34. Briolay A, Jaafar R, Nemoz G, Bessueille L. Myogenic differentiation and lipid-raft composition of $L 6$ skeletal muscle cells are modulated by PUFAs. Biochim Biophys Acta Biomembr. 2013;1828(2):602-13.

35. Fernandez A, Huggins IJ, Perna L, Brafman D, Lu D, Yao S, Gaasterland T, Carson DA, Willert K. The WNT receptor FZD7 is required for maintenance of the pluripotent state in human embryonic stem cells. Proc Natl Acad Sci. 2014;111(4):1409-14.

36. Price FD, Yin H, Jones A, van ljcken W, Grosveld F, Rudnicki MA. Canonical Wnt signaling induces a primitive endoderm metastable state in mouse embryonic stem cells. Stem Cells. 2013;31(4):752-64.

37. Huang TS, Li L, Moalim-Nour L, Jia D, Bai J, Yao Z, Bennett SA, Figeys D, Wang L. A regulatory network involving $\beta$-catenin, e-cadherin, PI3k/Akt, and slug balances self-renewal and differentiation of human pluripotent stem cells in response to Wnt signaling. Stem cells. 2015;33(5):1419-33.

38. Sokol SY. Maintaining embryonic stem cell pluripotency with Wnt signaling. Development. 2011;138(20):4341-50.

39. Davidson KC, Adams AM, Goodson JM, McDonald CE, Potter JC, Berndt JD, Biechele TL, Taylor RJ, Moon RT. Wnt/ $\beta$-catenin signaling promotes differentiation, not self-renewal, of human embryonic stem cells and is repressed by Oct4. Proc Natl Acad Sci. 2012;109(12):4485-90.

40. Famili F, Brugman MH, Taskesen E, Naber BE, Fodde R, Staal FJ. High levels of canonical Wnt signaling lead to loss of stemness and increased differentiation in hematopoietic stem cells. Stem Cell Rep. 2016;6(5):652-9.

41. Zheng B, Jarugumilli GK, Chen B, Wu X. Chemical probes to directly profile palmitoleoylation of proteins. ChemBioChem. 2016;17(21):202.

42. Kelly OG, Pinson KI, Skarnes WC. The Wnt co-receptors Lrp5 and Lrp6 are essential for gastrulation in mice. Development. 2004;131(12):2803-15.

43. Gadue P, Huber TL, Paddison PJ, Keller GM. Wnt and TGF- $\beta$ signaling are required for the induction of an in vitro model of primitive streak formation using embryonic stem cells. Proc Natl Acad Sci. 2006;103(45):16806-11.

44. Kubo A, Shinozaki K, Shannon JM, Kouskoff V, Kennedy M, Woo S, Fehling $\mathrm{HJ}$, Keller G. Development of definitive endoderm from embryonic stem cells in culture. Development. 2004;131(7):1651-62.

\section{Publisher's Note}

Springer Nature remains neutral with regard to jurisdictional claims in published maps and institutional affiliations.

Ready to submit your research? Choose BMC and benefit from

- fast, convenient online submission

- thorough peer review by experienced researchers in your field

- rapid publication on acceptance

- support for research data, including large and complex data types

- gold Open Access which fosters wider collaboration and increased citations

- maximum visibility for your research: over $100 \mathrm{M}$ website views per year

At $\mathrm{BMC}$, research is always in progress.

Learn more biomedcentral.com/submissions 